\title{
Silicane and germanane: tight-binding and first-principles studies
}

\author{
V. Zólyomi, J. R. Wallbank, and V. I. Fal'ko \\ Physics Department, Lancaster University, Lancaster LA1 4YB, United Kingdom \\ E-mail: v.zolyomi@lancaster.ac.uk
}

\begin{abstract}
We present a first-principles and tight-binding model study of silicane and germanane, the hydrogenated derivatives of two-dimensional silicene and germanene. We find that the materials are stable in freestanding form, analyse the orbital composition, and derive a tight-binding model using first-principles calculations to fit the parameters.
\end{abstract}

Submitted to: 2D Materials

PACS numbers: 73.20.-r, 71.15.Mb 


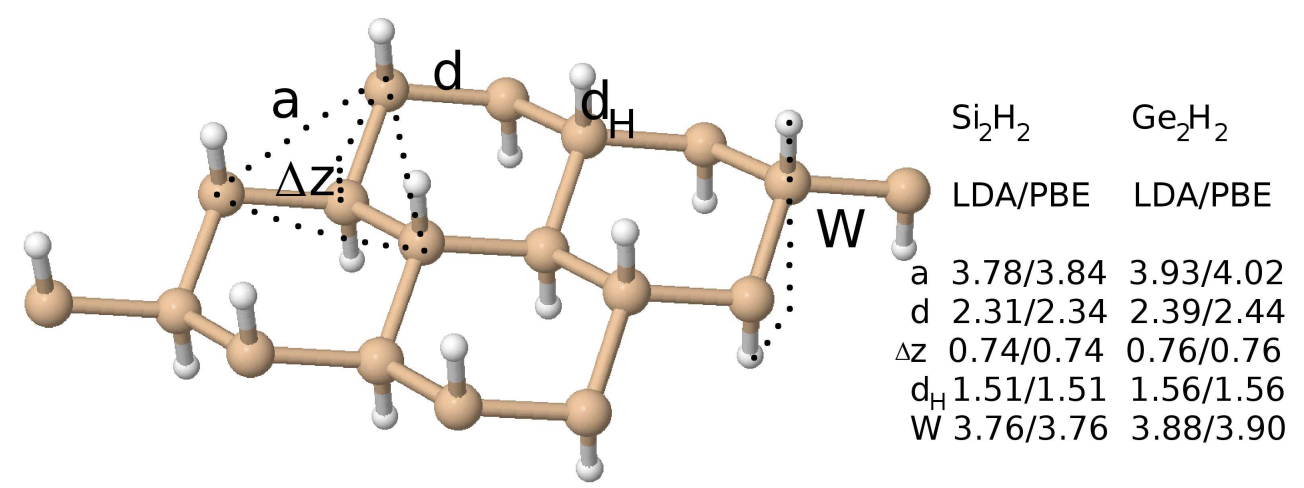

Figure 1. Structure of silicane and germanane. The larger spheres denote $\mathrm{Si} / \mathrm{Ge}$ atoms while the smaller ones denote hydrogens. Here $a$ is the lattice constant, $d$ the bond length between neighbouring $\mathrm{Si} / \mathrm{Ge}$ atoms, $\Delta z$ is the sublattice buckling between the $\mathrm{A}$ and B sublattice, $d_{H}$ is the distance between $\mathrm{Si} / \mathrm{Ge}$ and the hydrogen, and $W$ is the total width of the layer (excluding van der Waals radii of hydrogens). The structural paremeters are given in units of $\AA$.

Recent years have seen a rapid rise in research interest towards atomically thin twodimensional (2D) materials. Graphene has been in the focus of intensive research since its discovery [1,2, followed by silicene, $\mathrm{Si}_{2}$ [3] 6 . Practical application of graphene in nanoelectronics is somewhat limited by the lack of a band gap, and while alternatives such as hexagonal boron nitride [7, 8, and transition metal dichalcogenides [9 15 exist and others have been predicted theoretically such as gallium chalcogenides [16, significant research effort has been put into engineering a band gap in graphene. Complete hydrogenation was shown to be another way to engineer a band gap in graphene. The resulting material, graphane [17 $\left(\mathrm{C}_{2} \mathrm{H}_{2}\right)$ has a buckled honeycomb structure with a single hydrogen atom attached to each carbon site on alternating sides of the sheet. A recent experiment [18] has shown that few-layer germanane $\left(\mathrm{Ge}_{2} \mathrm{H}_{2}\right)$, the hydrogenated germanene, can be synthesised, expanding the family of atomic 2D materials.

In this work we provide a first-principles study of silicane and germanane. We present the phonon dispersions to illustrate that these materials are dynamically stable. The band structures, effective masses, charge carriers, and an orbital decomposition of the valence and conduction bands at high symmetry points in the Brillouin zone are presented. The latter information is utilised for building a tight-binding model for the description of the valence and conduction bands of silicane and germanane.

(I) Silicane and germanane have a honeycomb lattice as depicted in Fig. 1 The unit cell comprises two $\mathrm{Si} / \mathrm{Ge}$ atoms and two $\mathrm{H}$ atoms, and the $\mathrm{A}$ and $\mathrm{B}$ sublattices of $\mathrm{Si} / \mathrm{Ge}$ atoms exhibit a buckling. The tight-binding model we employ is an all-valence second-nearest neighbour model that takes into account four (one $s$ and three $p$ ) electrons per $\mathrm{Si} / \mathrm{Ge}$ atom and the single electron of hydrogen. The tight-binding Hamiltonian is

$$
\begin{aligned}
H= & H_{0}+H_{1}+H_{2} \\
H_{0}= & \sum_{i}\left(\varepsilon_{s} a_{i}^{+} a_{i}+\varepsilon_{p} \sum_{\alpha}\left(b_{i \alpha}^{+} b_{i \alpha}\right)+\varepsilon_{s} c_{i}^{+} c_{i}\right) \\
H_{1}= & \sum_{i}\left(\gamma_{s} c_{s} c_{i}^{+} a_{i}\right)+\gamma_{s s} a_{A}^{+} a_{B}+\sum_{\alpha}\left(\gamma_{s p} v_{\alpha}^{A B} a_{A}^{+} b_{B \alpha}+\gamma_{p p \sigma}\left(v_{\alpha}^{A B}\right)^{2} b_{A \alpha}^{+} b_{B \alpha}+\gamma_{p p \pi}(1-\right. \\
& \left.\left.\left(v_{\alpha}^{A B}\right)^{2}\right) b_{A \alpha}^{+} b_{B \alpha}\right)+\sum_{\alpha \neq \beta}\left(\gamma_{p p \sigma} v_{\alpha}^{A B} v_{\beta}^{A B} b_{A \alpha}^{+} b_{B \beta}-\gamma_{p p \pi} v_{\alpha}^{A B} v_{\beta}^{A B} b_{A \alpha}^{+} b_{B \beta}\right)+ \\
& \sum_{\alpha} \gamma_{s s^{H} p} v_{\alpha}^{A B} c_{A}^{+} b_{B \alpha}+h . c . \\
H_{2}= & \sum_{i}\left(\gamma_{s s}^{\prime} a_{i}^{+} a_{i^{\prime}}+\sum_{\alpha}\left(\gamma_{s p}^{\prime} v_{\alpha}^{A A^{\prime}} a_{i}^{+} b_{i^{\prime} \alpha}+\gamma_{p p \sigma}^{\prime}\left(v_{\alpha}^{A A^{\prime}}\right)^{2} b_{A \alpha}^{+} b_{A^{\prime} \alpha}+\gamma_{p p \pi}^{\prime}(1-\right.\right. \\
& \left.\left.\left(v_{\alpha}^{A A^{\prime}}\right)^{2}\right) b_{A \alpha}^{+} b_{A^{\prime} \alpha}\right)+\sum_{\alpha \neq \beta}\left(\gamma_{p p \sigma}^{\prime} v_{\alpha}^{A A^{\prime}} v_{\beta}^{A A^{\prime}} b_{A \alpha}^{+} b_{A^{\prime} \beta}-\gamma_{p p \pi}^{\prime} v_{\alpha}^{A A^{\prime}} v_{\beta}^{A A^{\prime}} b_{A \alpha}^{+} b_{A^{\prime} \beta}\right)+ \\
& \gamma_{s^{\prime} s_{s}}^{+} c_{A}^{+} a_{B}+\sum_{\alpha} \gamma_{s}^{\prime} v_{\alpha}^{A B^{\prime}} c_{A}^{+} b_{B \alpha}+h . c . \\
v_{\alpha}^{A B}= & \left(\mathbf{R}_{S i / G e}^{A}-\mathbf{R}_{S i / G e}^{B}\right)_{\alpha} /\left|\mathbf{R}_{S i / G e}^{A}-\mathbf{R}_{S i / G e}^{B}\right| \\
v_{\alpha}^{A A^{\prime}}= & \left(\mathbf{R}_{S i / G e}^{A}-\mathbf{R}_{S i / G e}^{A^{\prime}}\right) /\left|\mathbf{R}_{S i / G e}^{A}-\mathbf{R}_{S i / G e}^{A^{\prime}}\right|, v_{\alpha}^{A B^{\prime}}=\left(\mathbf{R}_{S i / G e}^{A}-\mathbf{R}_{H}^{B^{\prime}}\right)_{\alpha} /\left|\mathbf{R}_{S i / G e}^{A}-\mathbf{R}_{H}^{B^{\prime}}\right|
\end{aligned}
$$

Here, $a^{+}$and $a$ are the creation and annihilation operators of the $s$ electrons of $\mathrm{Si} / \mathrm{Ge}, b^{+}$and 
$b$ are the same for the $p$ electrons of $\mathrm{Si} / \mathrm{Ge}$, while $c^{+}$and $c$ are the same for the electrons of the $\mathrm{H}$ atoms. In $H_{0}$, parameters $\varepsilon_{s}$ and $\varepsilon_{p}$ are the on-site energies of the $s$ and $p$ orbitals of $\mathrm{Si} / \mathrm{Ge}$, $\varepsilon_{s^{H}}$ is the on-site energy of the electron of the hydrogen atom. In $H_{1}, \gamma_{s^{H}} s$ parameterises the nearest neighbour hopping between $s$ orbitals of Si/Ge and hydrogen, while $\gamma_{s s}, \gamma_{s p}, \gamma_{p p \sigma}$, and $\gamma_{p p \pi}$ are the nearest neighbour hoppings between $\mathrm{Si} / \mathrm{Ge}$ electrons on sublattice $\mathrm{A}$ and sublattice B. In $H_{2}$, parameters $\gamma_{s s}^{\prime}, \gamma_{s p}^{\prime}, \gamma_{p p \sigma}^{\prime}$, and $\gamma_{p p \pi}^{\prime}$ are the second-nearest neighbour hoppings between the Si/Ge electrons on the same sublattice, while $\gamma_{s^{H} s}^{\prime}$ and $\gamma_{s^{H} p}^{\prime}$ are the second-nearest neighbour hoppings between the orbitals of $\mathrm{Si} / \mathrm{Ge}$ and hydrogen on different sublattices. Summations in $i$ go over the $\mathrm{A}$ and $\mathrm{B}$ sublattices while summations in $\alpha$ and $\beta$ go over $x, y, z ; v_{\alpha}^{A B}, v_{\alpha}^{A A^{\prime}}$, and $v_{\alpha}^{A B^{\prime}}$ take into account the orientation of the $p$ orbitals, where $\mathbf{R}$ denotes the coordinates of the atoms. The Hamiltonian in the Slater-Koster approach [19], shown with solid lines separating A and B sublattice contributions as well as hydrogen contributions has the form of a $10 \times 10$ matrix:

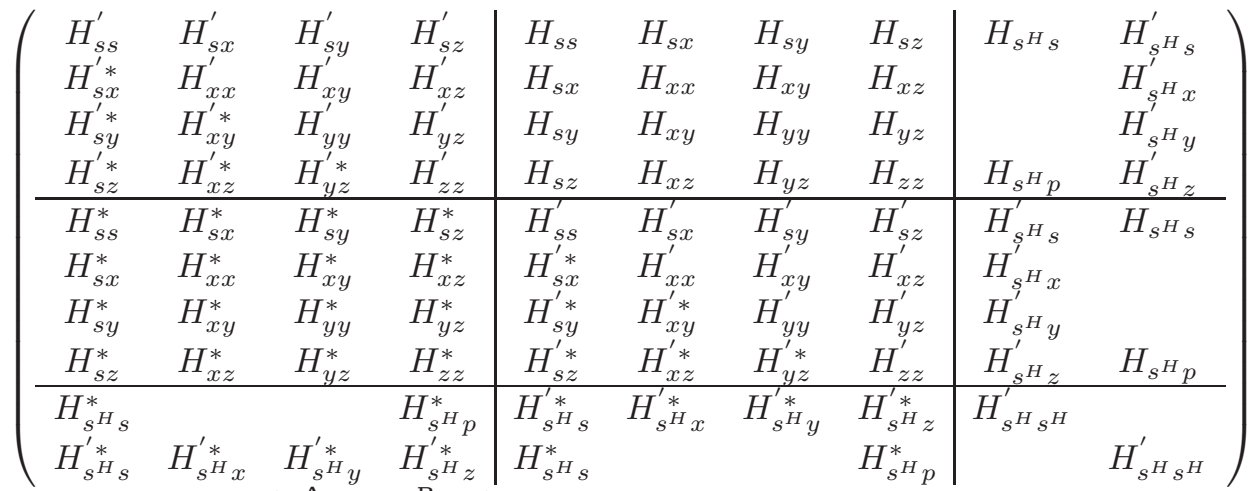

$$
\begin{aligned}
& H_{s s}=\gamma_{s s} \sum_{B} e^{i \mathbf{k}\left(\mathbf{R}_{\mathbf{S i} / \mathbf{G e}^{\mathrm{e}}}^{\mathbf{A}}-\mathbf{R}_{\mathrm{Si} / \mathbf{G e}}^{\mathrm{B}}\right)}, H_{s^{H} s}=\gamma_{s^{H} s}, H_{s^{H} p}=\gamma_{s^{H}} p \\
& H_{s \alpha}=\gamma_{s p} \sum_{B} v_{\alpha}^{A B} e^{i \mathbf{k}\left(\mathbf{R}_{\mathbf{S i} / \mathbf{G e}}^{\mathrm{A}}-\mathbf{R}_{\mathrm{Si} / \mathbf{G e}}^{\mathrm{B}}\right)}, H_{s \alpha}^{\prime}=\gamma_{s p}^{\prime} \sum_{A} v_{\alpha}^{A A^{\prime}} e^{i \mathbf{k}\left(\mathbf{R}_{\mathbf{S i} / \mathbf{G e}}^{\mathbf{A}}-\mathbf{R}_{\mathbf{S i} / \mathbf{G e}}^{\mathbf{A}^{\prime}}\right)}
\end{aligned}
$$

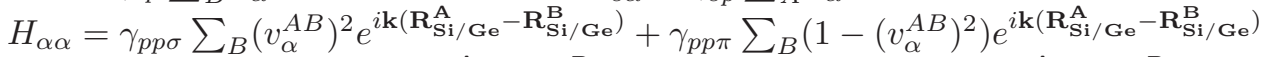

$$
\begin{aligned}
& H_{\alpha \beta}=\gamma_{p p \sigma} \sum_{B} v_{\alpha}^{A B} v_{\beta}^{A B} e^{i \mathbf{k}\left(\mathbf{R}_{\mathbf{S i} / \mathbf{G e}}^{\mathbf{A}}-\mathbf{R}_{\mathbf{S i} / \mathbf{G e}}^{\mathrm{B}}\right)}-\gamma_{p p \pi} \sum_{B} v_{\alpha}^{A B} v_{\beta}^{A B} e^{i \mathbf{k}\left(\mathbf{R}_{\mathbf{S i} / \mathbf{G e}}^{\mathbf{A}}-\mathbf{R}_{\mathbf{S i} / \mathbf{G e}}^{\mathrm{B}}\right)}, \alpha \neq \beta \\
& H_{\alpha \alpha}^{\prime}=\varepsilon_{p}+\gamma_{p p \sigma}^{\prime} \sum_{A}\left(v_{\alpha}^{A A^{\prime}}\right)^{2} e^{i \mathbf{k}\left(\mathbf{R}_{\mathbf{S i} / \mathbf{G e}}^{\mathbf{A}}-\mathbf{R}_{\mathbf{S}^{\prime} / \mathbf{G e}}^{\mathbf{A}^{\prime}}\right)}+\gamma_{p p \pi}^{\prime} \sum_{A}\left(1-\left(v_{\alpha}^{A A^{\prime}}\right)^{2}\right) e^{i \mathbf{k}\left(\mathbf{R}_{\mathbf{S i} / \mathbf{G e}}^{\mathbf{A}}-\mathbf{R}_{\mathbf{S i}}^{\mathbf{A}^{\prime} / \mathbf{G e}}\right)} \\
& H_{\alpha \beta}^{\prime}=\gamma_{p p \sigma}^{\prime} \sum_{A} v_{\alpha}^{A A^{\prime}} v_{\beta}^{A A^{\prime}} e^{i \mathbf{k}\left(\mathbf{R}_{\mathrm{Si} / \mathrm{Ge}}^{\mathbf{A}}-\mathbf{R}_{\mathbf{S i} / \mathbf{G e}}^{\mathbf{A}^{\prime}}\right)}-\gamma_{p p \pi}^{\prime} \sum_{A} v_{\alpha}^{A A^{\prime}} v_{\beta}^{A A^{\prime}} e^{i \mathbf{k}\left(\mathbf{R}_{\mathrm{Si} / \mathbf{G e}}^{\mathbf{A}}-\mathbf{R}_{\mathbf{S i} / \mathbf{G e}}^{\mathbf{A}^{\prime}}\right)}, \alpha \neq \beta \\
& H_{s^{H} s}^{\prime}=\gamma_{s^{H} s}^{\prime} \sum_{B} e^{i \mathbf{k}\left(\mathbf{R}_{\mathbf{S i} / \mathbf{G e}^{\mathbf{A}}}^{\mathbf{A}}-\mathbf{R}_{\mathbf{H}}^{\mathbf{B}^{\prime}}\right)}, H_{s^{H} s^{H}}^{\prime}=\varepsilon_{s}^{H}+\gamma_{s^{H} s^{H}}^{\prime} \sum_{A} e^{i \mathbf{k}\left(\mathbf{R}_{\mathbf{S i} / \mathbf{G e}^{\mathbf{A}}}^{\mathbf{A}}-\mathbf{R}_{\mathbf{S}}^{\mathbf{A}^{\prime} / \mathbf{G e}}\right)} \\
& H_{s^{H} \alpha}^{\prime}=\gamma_{s^{H} p}^{\prime} \sum_{B} v_{\alpha}^{A B^{\prime}} e^{i \mathbf{k}\left(\mathbf{R}_{\mathbf{S}}^{\mathbf{A}} / \mathbf{G e}^{-}-\mathbf{R}_{\mathbf{H}}^{\mathbf{B}^{\prime}}\right)}, H_{s s}^{\prime}=\varepsilon_{s}+\gamma_{s s}^{\prime} \sum_{A} e^{i \mathbf{k}\left(\mathbf{R}_{\mathbf{S i}}^{\mathbf{A}} / \mathbf{G e}^{-}-\mathbf{R}_{\mathbf{S}}^{\mathbf{A}^{\prime} / \mathbf{G e}}\right)}
\end{aligned}
$$

The total number of parameters in this model is sixteen, but one can choose one of the on-site energies to be zero to set the Fermi level leaving fifteen parameters to fit. The resulting model can be used to provide a simple semiempirical reproduction of first-principles band structures, as we will show below.

(II) The parameters in this model, as well as the justification for the choice of orbitals comes from first-principles studies using density functional theory (DFT) implemented in the VASP 20 plane-wave-basis code. First, we calculated the lattice parameters of silicane and germanane with multiple semilocal exchange-correlation functionals: the local density approximation (LDA) and the Perdew-Burke-Ernzerhof [21] (PBE) functionals. In addition the screened Heyd-ScuseriaErnzerhof 06 (HSE06) functional 22] was used to obtain the electronic band structures to compensate (at least partially) for the underestimation of the band gap by semilocal functionals. The plane-wave cutoff energy was $500 \mathrm{eV}$. A $12 \times 12$ Monkhorst-Pack k-point grid was used for geometry optimisations while a $24 \times 24$ grid was used to calculate the band structures. The vertical separation of periodic images of the monolayer was set to $15 \AA$. The force tolerance in the optimisation was $0.005 \mathrm{eV} / \AA$. Phonons were calculated with the force constant approach in a $3 \times 3$ supercell.

We find that the relaxed structure of both silicane and germanane is very similar to that of graphane, as illustrated in Fig. 1. The parameters listed were obtained after a full geometry 

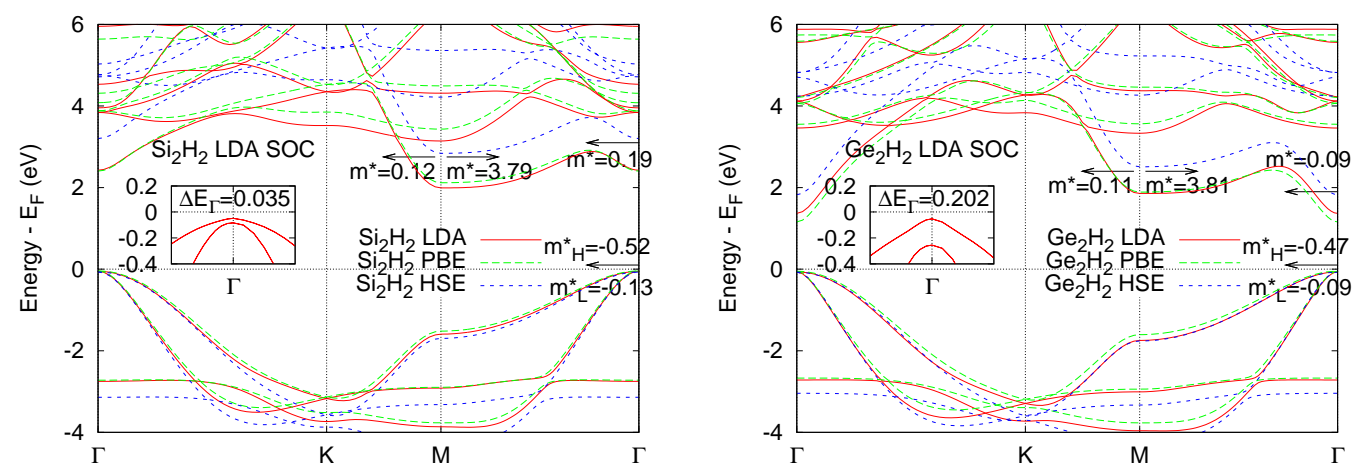

Figure 2. Band structures of silicane and germanane. The zero of energy is taken to be the Fermi level and the top of the valence band is marked with a horizontal line. The effect of spinorbit coupling at the $\Gamma$ point is illustrated in the insets. Effective masses (in units of electron mass) in the HSE06 calculations are provided in the conduction band at M and $\Gamma$, and in the valence band at $\Gamma$ (where the $H$ and $L$ subscript refers to the heavy and light effective mass). Our results on $\mathrm{Ge}_{2} \mathrm{H}_{2}$ compare well with Ref. [18. It is worth noting that we find almost no sign of anisotropy in the effective masses at $\Gamma$. In comparison to the literature on graphene, an LDA study found a small anisotropy in both the valence and conduction band of graphane 31, while an earlier GGA study makes no mention of any such anisotropy 29].

optimisation. The bond lengths obtained with the PBE functional are systematically larger than those optimised with the LDA, as expected [23. Note that the hydrogenation is accompanied by a significant increase in the magnitude of the sublattice buckling when compared to silicene and germanene, where, according to LDA/PBE, $\Delta z=0.44 / 0.45 \AA$ and $\Delta z=0.65 / 0.69 \AA$, respectively. Lattice constants and sublattice bucklings agree with previous literature within $\pm 5 \%$ and $\pm 10 \%$, respectively 24]28.

The calculated electronic band structures are plotted in Fig. 2. In comparison to graphane 29] one important difference in silicane and germanane is that in the latter two materials a band appears close to the conduction band edge at the $\mathrm{M}$ point. While the conduction band minimum of $\mathrm{Ge}_{2} \mathrm{H}_{2}$ is at the $\Gamma$ point similar to $\mathrm{C}_{2} \mathrm{H}_{2}$, in the case of $\mathrm{Si}_{2} \mathrm{H}_{2}$ it is in fact at the $\mathrm{M}$ point, making silicane an indirect gap semiconductor. The band gaps of silicane and germanane are $2.91 \mathrm{eV}$ and $1.90 \mathrm{eV}$, respectively, according to the HSE06 functional which is expected to underestimate the gap by no more than $10 \%$ 30. Note that the conduction band is anisotropic at the $\mathrm{M}$ point with a heavy effective mass in the $\Gamma$ direction. Our finding that the band gap of $\mathrm{Si}_{2} \mathrm{H}_{2}$ is indirect and that of $\mathrm{Ge}_{2} \mathrm{H}_{2}$ is direct is supported by previous literature using a variety of methods ranging from semi-local DFT through hybrid functionals to single-shot GW 24, 25, 27, 28.

We have also looked at the effects of spin-orbit coupling. The insets in Fig. 2 show the spin-orbit induced splitting of the valence band at the $\Gamma$ point. The effect of spin-orbit coupling is more pronounced in the case of germanane where the valence band splits by an energy difference of $202 \mathrm{meV}$ at $\Gamma$.

Now we discuss the orbital composition of the valence and conduction bands of silicane and germanane (see Table 1). We find that at the $\Gamma$ point the valence band consists of $\mathrm{Si} / \mathrm{Ge} p_{x}$ and $p_{y}$ orbitals, while the conduction band is dominantly $\mathrm{Si} / \mathrm{Ge} s$ and $p_{z}$. However, at the M point the conduction band also contains $\mathrm{Si} / \mathrm{Ge} p_{x}$ and $p_{y}$ contributions. The $\mathrm{H} s$ orbital also contributes at the $\mathrm{M}$ point, to the valence band in silicane (and slightly to the conduction band in germanane). This means that for a tight-binding description of silicane and germanane an all valence description is required taking into account the $s, p_{x}, p_{y}$, and $p_{z}$ orbitals of $\mathrm{Si} / \mathrm{Ge}$, as well as the $\mathrm{H} s$ orbital.

(III) We used the HSE06 band structures as reference to obtain the tight-binding parameters. We find that the tight-binding band structure can reproduce the entirety of the DFT valence band and the vicinity of the conduction band at both the $\Gamma$ and $\mathrm{M}$ points (see Fig. 31). It is important to note here that if we neglect second-nearest neighbour interactions the valence band can still be 
Silicane and germanane: tight-binding and first-principles studies

Table 1. Orbital decomposition of the valence and conduction bands of silicane and germanane at the $\Gamma$ and $\mathrm{M}$ points according to the local density approximation.

\begin{tabular}{lcc}
\hline & $\Gamma$ & $\mathrm{M}$ \\
\hline $\mathrm{Si}_{2} \mathrm{H}_{2}$ val. & $0.23\left(p_{x}^{S i}+p_{y}^{S i}\right)$ & $0.05 p_{x}^{S i}+0.16 p_{y}^{S i}$ \\
$\mathrm{Si}_{2} \mathrm{H}_{2}$ cond. & $0.09 s^{S i}+0.05 p_{z}^{S i}+0.03 s^{H}$ & $0.07 s^{S i}+0.01 p_{x}^{S i}+0.01 p_{y}^{S i}+0.03 p_{z}^{S i}$ \\
$\mathrm{Ge}_{2} \mathrm{H}_{2}$ val. & $0.32\left(p_{x}^{G e}+p_{y}^{G e}\right)$ & $0.07 p_{x}^{G e}+0.22 p_{y}^{G e}$ \\
$\mathrm{Ge}_{2} \mathrm{H}_{2}$ cond. & $0.24 s^{G e}+0.06 p_{z}^{G e}$ & $0.11 s^{G e}+0.03 p_{x}^{G e}+0.03 p_{z}^{G e}$ \\
\hline
\end{tabular}
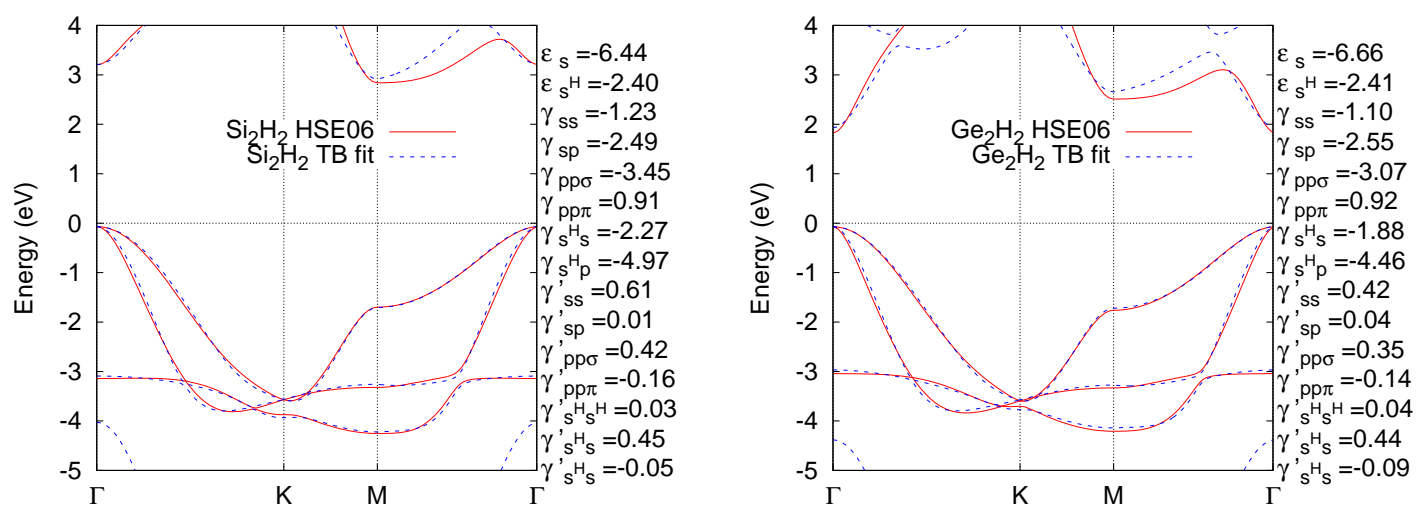

Figure 3. Tight-binding band structures of silicane and germanane compared with the HSE06 DFT bands. The parameters of the model are shown in the legend in units of $\mathrm{eV}$. The reference energy level is set by $\varepsilon_{p}=0$.

reproduced but the behaviour of the conduction band at the $\mathrm{M}$ point cannot, which indicates that the second nearest neighbour interactions are responsible for the minimum in the conduction band at the M point. Also, the $d$-shell of $\mathrm{Si} / \mathrm{Ge}$ is likely to affect states in the conduction band. The best fit is achieved with the parameters listed in the legend of Fig. 3. the fitting was optimised to give a quantitative description of the valence band and the conduction band near the $\Gamma$ and the M point.

(IV) Finally, we have performed a full geometry optimisation of $\mathrm{Si}_{2} \mathrm{H}_{2}$ and $\mathrm{Ge}_{2} \mathrm{H}_{2}$. While the geometry optimisation yields an energetically stable configuration for both materials, it is necessary to examine their phonon dispersions in order to ascertain whether they are dynamically stable. We find that silicane is stable as there is no sign of any dynamical instability anywhere along the high symmetry lines of the Brillouin zone (see Fig. 4). In the case of $\mathrm{Ge}_{2} \mathrm{H}_{2}$ we find a small pocket of instability for the flexural acoustic phonons. We believe that this is an artifact arising due to the difficulty in converging the flexural acoustic branch of two-dimensional materials as the phonon wave vector goes to zero. Since we find no other pockets of instability in any of the other branches we conclude that germanane is also dynamically stable. This is an important finding as the experiments in Ref. [18] were performed on multilayers of germanane on a substrate, while our calculations predict that suspended single-layer germanane would be stable, too.

It is worth noting that there is an alternate configuration for silicane and germanane not considered here. The so-called chair-like structure we studied corresponds to the case when $\mathrm{H}$ atoms alternate on the two sides of the sheet such that for each sublattice the $\mathrm{H}$ atom is on a fixed side. In the so-called boat configuration the $\mathrm{H}$ atoms alternate in pairs instead, which slightly increases the unit cell size. The latter has been shown to be notably less stable than the chair configuration in the case of graphane 29, nevertheless the boat configuration of silicane and germanane has been found to be stable 25] which is important to bear in mind.

In conclusion we have shown using first-principles density functional theory that freestanding hydrogenated silicene and germanene, better known as silicane and germanane, are energetically 

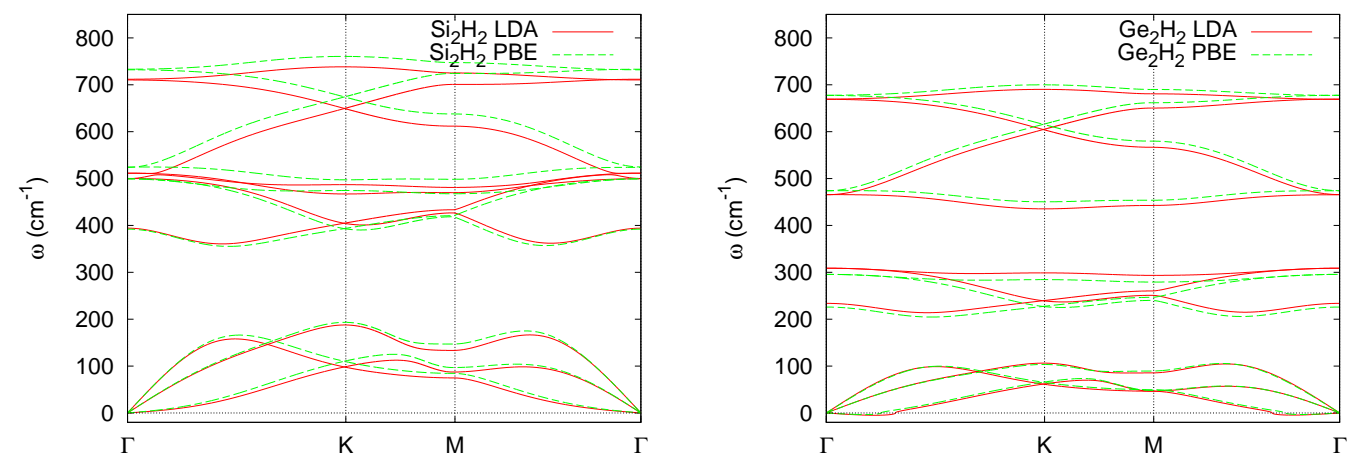

Figure 4. Phonon dispersions of silicane and germanane. The high frequency branch above $2000 \mathrm{~cm}^{-1}$ corresponding to $\mathrm{Si}-\mathrm{H} / \mathrm{Ge}-\mathrm{H}$ vibrations is omitted for sake of clarity.

and dynamically stable. We have shown that silicane is an indirect and germanane a direct gap semiconductor and derived a tight binding model to describe the valence and conduction bands of these materials, fitting the parameters to hybrid density functional calculations. A minimum in the conduction band at the M point is caused by second nearest neighbour interaction which, in the case of silicane, leads to an indirect band gap.

\section{Acknowledgments}

We acknowledge financial support from EC-FET European Graphene Flagship Project, EPSRC Science and Innovation Award, ERC Synergy Grant "Hetero2D," the Royal Society Wolfson Merit Award, and the Marie Curie project CARBOTRON.

\section{References}

[1] K.S. Novoselov, A.K. Geim, S.V. Morozov, D. Jiang, Y. Zhang, S.V. Dubonos, I.V. Grigorieva, and A.A. Firsov, Science 306, 666 (2004).

[2] A.K. Geim and K.S. Novoselov, Nature Materials 6, 183 (2007).

[3] B. Aufray, A. Kara, S. Vizzini, H. Oughaddou, C. Landri, B. Ealet, and G. L. Lay, Appl. Phys. Lett. 96, $183102(2010)$.

[4] P. E. Padova, C. Quaresima, C. Ottaviani, P. M. Sheverdyaeva, P. Moras, C. Carbone, D. Topwal, B. Olivieri, A. Kara, H. Oughaddou, B. Aufray, and G. L. Lay, Appl. Phys. Lett. 96, 261905 (2010).

[5] B. Lalmi, H. Oughaddou, H. Enriquez, A. Kara, S. Vizzini, B. Ealet, and B. Aufray, Appl. Phys. Lett. 97, 223109 (2010).

[6] N. D. Drummond, V. Zólyomi, and V. I. Fal'ko, Phys. Rev. B 85, 075423 (2012).

[7] Y. Kubota, K. Watanabe, O. Tsuda, and T. Taniguchi, Science 317, 932 (2007)

[8] M.P. Levendorf, C.-J. Kim,L. Brown, P.Y. Huang, R.W. Havener, D.A. Muller, and J. Park, Nature 488, 627 (2012).

[9] K.F. Mak, C. Lee, J. Hone, J. Shan, and T.F. Heinz, Phys. Rev. Lett. 105, 136805 (2010).

[10] B. Radisavljevic, A. Radenovic, J. Brivio, V. Giacometti, and A. Kis, Nature Nanotechnology 6, 147 (2011)

[11] B. Radisavljevic, M.B. Whitwick, and A. Kis, ACS Nano 5, 9934 (2011).

[12] T. Georgiou et al., Nature Nanotechnology 8, 100 (2013).

[13] J.N. Coleman et al., Science 331, 568 (2011).

[14] D. Braga, L.I. Gutirrez, H. Berger, and A.F. Morpurgo, Nano Lett. 12, 5218 (2012).

[15] C. Ataca, H. Sahin, and S. Ciraci, J. Phys. Chem. C 116, 8983 (2012).

[16] V. Zólyomi, N. D. Drummond, and V. I. Fal'ko, Phys. Rev. B 87, 195403 (2013).

[17] D. C. Elias, R. R. Nair, T. M. G. Mohiuddin, S. V. Morozov, P. Blake, M. P. Halsall, A. C. Ferrari, D. W. Boukhvalov, M. I. Katsnelson, A. K. Geim, and K. S. Novoselov, Science 323, 610 (2009).

[18] E. Bianco, S. Butler, S. Jiang, O. D. Restrepo, W. Windl, and J. E. Goldberger, ACS Nano 7, 4414 (2013).

[19] J. C. Slater and G. F. Koster, Physical Review 94, 1498 (1954).

[20] G. Kresse and J. Furthmüller, Phys. Rev. B 54, 11169 (1996).

[21] J. P. Perdew, K. Burke, and M. Ernzerhof, Phys. Rev. Lett. 77, 3865 (1996).

[22] J. Heyd, G.E. Scuseria, and M. Ernzerhof, J. Chem. Phys. 118, 8207 (2003); A.V. Krukau, O.A. Vydrov, A.F. Izmaylov, and G.E. Scuseria, ibid. 125, 224106 (2006). 
[23] F. Favot and A. D. Corso, Phys. Rev. B 60, 11427 (1999).

$[24]$ L. C. Lew Yan Voon, E. Sandberg, R. S. Aga, and A. A. Farajian, Appl. Phys. Lett. 97, 163114 (2010).

[25] M. Houssa, E. Scalise, K. Sankaran, G. Pourtois, V. V. Afanasev and A. Stesmans, Appl. Phys. Lett. 98 223107 (2011).

[26] J. C. Garcia, D. B. de Lima, L. V. C. Assali, and J. F. Justo, J. Phys. Chem. C 115, 13242 (2011).

[27] O. Pulci, P. Gori, M. Marsili, V. Garbuio, R. Del Sole, and F. Bechstedt, Eur. Phys. Lett. 98, 37004 (2012).

[28] W. Wei and T. Jacob, Phys. Rev. B 88, 045203 (2013).

[29] J. O. Sofo, A. S. Chaudhari, and G. D. Barber, Phys. Rev. B 75, 153401 (2007).

[30] S. Park, B. Lee, S. H. Jeon, and S. Han, Current Applied Physics 11, S337 (2011).

[31] L. Liu and Z. Shen, Appl. Phys. Lett. 95, 252104 (2009). 